\title{
Contribuição de cesta básica na segurança alimentar de idosos diabéticos de programa assistencial em uma cidade do sul do Brasil
}

\section{Contribation of thebasicfood besket tothentritiond stede of dabetic ddaly in an assistance programinasathem Brazliancty}

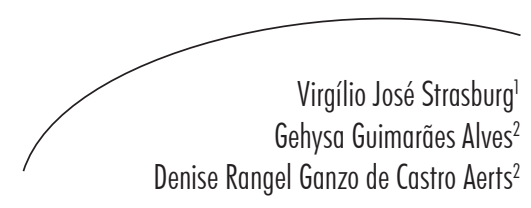

Resumo

A garantia de acesso em quantidade, qualidade e regularidade aos alimentos é direito constitucional e humano. Em função disso, os programas públicos de segurança alimentar procuram focar suas ações nas populações de maior vulnerabilidade social. Este trabalho teve como objetivo investigar a contribuição energética e de macronutrientes da composição da cesta básica diet, oferecida pelo Programa Cidadania Alimentar (PCA) da cidade de Canoas-RS, ao estado nutricional dos idosos diabéticos. Para tanto, foi realizado estudo descritivo incluindo os 163 idosos diabéticos, de ambos os sexos, cadastrados no PCA que receberam o benefício da cesta básica diet. Constatou-se que 76,1\% dos inscritos são do sexo feminino e 68,1\% apresentaram classificação do índice de massa corporal (IMC) $>27 \mathrm{~kg} / \mathrm{m}^{2}$, indicando excesso de peso. A composição da cesta básica diet fornecida pelo PCA contemplou a média de 924,4 kcal/dia, distribuída em 54,2\% de carboidratos, $13,4 \%$ de proteínas e $32,5 \%$ de gorduras. Os resultados mostraram boa adequação energética da cesta básica diet quando considerada a necessidade energética estimada (NEE) recomendada para os idosos. No entanto, sugere-se uma reavaliação do programa para que ele possa atuar em ações promotoras de saúde frente aos resultados do IMC encontrados nessa população.

\section{Abstract}

Ensuring access in quantity, quality and regularity to foods is a constitutional and human right. As a result, public food safety programs seek to focus their efforts on the most socially vulnerable populations. The main objective of this work was to investigate the energetic contribution and macronutrients composition for the diet food basket offered by Programa Cidadania Alimentar (PCA)* from the city of Canoas to the nutritional state of diabetic elders. A descriptive study was conducted, including 163 diabetic elderly of both genders enrolled in PCA, who received the benefit of a diet

Palavras-chave: Avaliação nutricional. Diabetes Mellitus. Idoso. Cesta básica. Segurança alimentar nutricional. Programas Governamentais.
Key words: Nutritional status. Diabetes Mellitus. Elderly. Basic food basket. Food and Nutrition Security. Government Programs.

\footnotetext{
Departamento de Medicina Social, Graduação de Nutrição. Universidade Federal do Rio Grande do Sul. Porto Alegre, RS, Brasil.

2 Programa de Pós-graduação em Saúde Coletiva. Universidade Luterana do Brasil. Canoas, RS, Brasil.
}

Correspondência / Correspondence

Virgílio José Strasburg

E-mail: virgilio_nut@ufrgs.br 
package food. It was perceived that $76.1 \%$ of the enrolled participants are female, $68.1 \%$ showed body mass index (BMI) classification of $>27 \mathrm{~kg} / \mathrm{m}^{2}$, indicating overweight. The diet food basket composition supplied by the PCA includes the average of $924.4 \mathrm{kcal} /$ day, distributed in $54.2 \%$ of carbohydrates, $13.4 \%$ of proteins and $32.5 \%$ of fats. The results showed good energetic adequacy of a diet food basket when the estimate energy requirements (ERR) for elders is taken into account. However it is suggested a program reevaluation in order to promote health acts considering BMI found in this population.

* Programa Cidadania Alimentar (Food Citizenship Program) is an assistance program conducted in Canoas city (Brazil).

\section{INTRODUÇÃO}

A garantia de alimentação para cada ser humano, além de ser um determinante de saúde, é condição básica para o direito à vida e o exercício da cidadania. ${ }^{1}$ Os objetivos dos programas públicos de acesso a alimentação adequada procuram focar suas ações nas populações em situação de maior vulnerabilidade social, ${ }^{2}$ dentre as quais se destacam os idosos.

A população de idosos no país vem apresentando um crescimento constante. Estudos de projeção estatística apontam que o Brasil deverá ter a sexta maior população de idosos do mundo. Estima-se que, no ano de 2025, sejam em torno de $15 \%$ da população total do país, com um contingente aproximado de 32 milhões de pessoas. ${ }^{3} \mathrm{O}$ acréscimo a essa expectativa de vida passou a ser uma resposta à mudança de alguns indicadores de saúde associados, nos quais estão relacionadas as condições sociais, econômicas, geográficas, de padrões de consumo, os estilos de vida e urbanização acelerada. ${ }^{4}$

Essa transição demográfica trouxe consigo uma nova situação epidemiológica, na qual prevaleceram as doenças crônicas, denominadas como doenças e agravos não-transmissíveis (DANTs). Essas são caracterizadas como de início gradual, de duração longa, e de causas múltiplas, dentre as quais podem ser incluídas a obesidade, o diabetes mellitus, a hipertensão arterial, as doenças cardíacas e os cânceres. ${ }^{5}$ A Organização
Mundial da Saúde (OMS) estima que, até o ano de 2020, as DANTs serão responsáveis por $78 \%$ da carga global das doenças nos países em desenvolvimento. ${ }^{6}$

Entre as DANTs, o diabetes mellitus (DM) afeta, atualmente, cerca de $6 \%$ da população adulta no mundo, sendo que $80 \%$ destes estão nos países em desenvolvimento, de acordo com o International Diabetes Federation (IDF). ${ }^{7}$ No Brasil, esse número deverá chegar a mais de 11,3 milhões até o ano de $2030 .{ }^{8}$ Sua incidência é crescente, aumentando com a idade e atingindo, na atualidade, proporções epidêmicas que demandam alto custo econômico e social. Essa é uma causa importante de mortalidade em função da elevada frequência de casos de complicações associadas, nas quais se destacam insuficiência renal, amputação de membros inferiores, cegueira e doença cardiovascular.' Trabalhar em ações que contribuam com a qualidade de vida dos diabéticos é um desafio constante, sobretudo quando estão associados aos fatores de idade e vulnerabilidade social.

No ano de 2003, foi implantado o Programa Cidadania Alimentar (PCA), na cidade de Canoas no Rio Grande do Sul (RS), o qual distribuía uma cesta básica padrão com o objetivo de suprir $50 \%$ das necessidades energéticas dos idosos de baixa renda, ou seja, para os que tinham rendimento equivalente a um salário mínimo. Atualmente, o programa possui, além da composição padrão, outras especificações para atender às necessidades 
alimentares encontradas entre a população idosa: light, composta por alimentos com teor reduzido de lipídeos para pessoas que estão acima do peso e/ou com alto índice de colesterol e hipertensão, e diet, direcionada às pessoas com diabetes, que será o objeto deste estudo. Os termos light e diet foram nomenclaturas específicas criadas pelo PCA, bem como a relação dos alimentos e suas quantidades para cada composição de cesta básica.

Considerando o fato de não haver políticas públicas federais ou estaduais que contemplem as condições de acesso à alimentação para esse grupo, o objetivo do presente estudo foi investigar a contribuição energética e de macronutrientes da composição da cesta básica diet, oferecida pelo Programa Cidadania Alimentar (PCA) da cidade de Canoas/RS, ao estado nutricional dos idosos diabéticos.

\section{METODOLOGIA}

Foi realizado estudo descritivo com dados secundários extraídos dos prontuários do Programa Cidadania Alimentar (PCA) junto à Secretaria Municipal de Assistência Social e Cidadania (SMASCI) da cidade de Canoas (RS). Essa é a cidade mais populosa da Região Metropolitana de Porto Alegre, com 326.458 habitantes, e a expectativa de vida de sua população é de 71,4 anos. ${ }^{10}$

A população deste estudo foi composta por 163 idosos com 60 anos ou mais, de ambos os sexos, diagnosticados nos prontuários do PCA como diabéticos. Foram coletados dados relacionados com as variáveis sexo, idade, peso, estatura e utilização de insulina e/ou de medicação hipoglicemiante. Também foram verificadas informações sociodemográficas que contemplaram o estado civil, a composição familiar e o tempo de cadastramento no PCA.

Devido às características de mobilidade desse programa social - isto é, a entrada e saída de beneficiários podem ocorrer em qualquer tempo
-, foram utilizadas como critério de inclusão do estudo as informações dos idosos que fizeram a retirada da cesta básica diet ( $\operatorname{cb}$ diet) no mês de julho de 2008. Essa amostra foi intencional, contemplando o meio do período do ano. Prontuários com informações inconsistentes ou incompletas de outros idosos diabéticos que não retiraram a cbdiet no mês de referência foram excluídos (oito casos).

A pesquisa teve a aprovação da SMASCI da cidade de Canoas / RS e do Comitê de Ética da Universidade Luterana do Brasil sob o $\mathrm{n}^{\circ}$ 2008-594H.

Quanto à idade, os dados dos idosos foram agrupados por estratificação etária: 60 a 69 anos; 70 a 79 anos; e acima de 80 anos. Foi obtido o índice de massa corporal (IMC) por meio do cálculo do peso atual do idoso, dividido pela sua altura (em metros) ao quadrado. Para a avaliação do estado nutricional dos idosos, foram utilizadas as recomendações de Lipschitz para as seguintes categorias de IMC: $<22 \mathrm{~kg} / \mathrm{m}^{2}$ para magreza; $>22$ e $<27 \mathrm{~kg} / \mathrm{m}^{2}$ para eutrofia; $>27$ e $<30 \mathrm{~kg} /$ $\mathrm{m}^{2}$ para sobrepeso. ${ }^{11}$ Além desses, também foi incluído o parâmetro para obesidade quando o IMC $>30 \mathrm{~kg} / \mathrm{m}^{2}$ de acordo com o padrão da OMS. ${ }^{12}$ Também foi realizado o cálculo da necessidade energética estimada (NEE) da população, com base nas equações propostas pelo Institute of Medicine (IOM): ${ }^{13}$

Homem: NEE $=662-(9,53 \times$ idade (anos)) + nível de atividade física $\mathrm{x}$ $[(15,91 \times$ peso $(\mathrm{kg}))+539,6 \times$ altura $(\mathrm{m}))]$.

Mulher: NEE $=354-(6,91 \times$ idade (anos)) + nível de atividade física $\mathrm{x}$ $[(9,36 \times$ peso $(\mathrm{kg}))+(726 \times$ altura $(\mathrm{m}))]$.

Para ambos os casos, foi utilizada a constante 1,0 (sedentária) para calcular o nível de atividade física e que contempla trabalhos domésticos de esforço leve a moderado e caminhadas para atividades relacionadas ao cotidiano. 
Os dados relacionados à composição alimentar foram verificados junto à documentação do PCA, enquanto as respectivas informações nutricionais dos produtos foram retiradas de uma amostra da cbdiet, numa entrega realizada pelo programa.

Para a cbdiet, foram calculados o valor energético total e a média diária per capita, com os respectivos percentuais dos nutrientes. Levando em consideração que as informações constantes nos rótulos dos alimentos são de responsabilidade do fabricante de cada um dos produtos, realizou-se, para efeito de validação das informações obtidas, comparativo sobre a informação nutricional. Nesse procedimento, foram utilizadas as tabelas de composição de alimentos do Instituto Brasileiro de Geografia e Estatística (IBGE) e TACO, do Núcleo de Estudos e Pesquisas em Alimentação (NEPA) da Universidade Estadual de Campinas (Unicamp). ${ }^{14,15}$ Nos produtos "adoçante à base de stévia" e "proteína texturizada de soja", foram utilizados os mesmos valores da rotulagem nutricional da embalagem dos alimentos, pelo fato destes não constarem nas respectivas tabelas.

A verificação do percentual de contribuição da cbdiet ao estado nutricional dos idosos diabéticos foi obtido pelo resultado do cálculo entre a média energética diária oferecida pelos alimentos e a mediana das NEEs (estratificadas para as faixas etárias em ambos os sexos dos beneficiados).

A codificação e a entrada de dados no programa Microsoft Excel $^{\circledR}$ foi realizada duas vezes, com o intuito de diminuir os erros de consistência. As variáveis quantitativas foram analisadas descritivamente com o cálculo de medianas e desvios-padrões e de análises estratificadas segundo as variáveis "sexo" e "faixa etária".

A análise estatística dos dados foi realizada no programa Statistical Package for the Social Sciences for Windows (SPSS) versão $10.0^{\circledR}$.

\section{RESULTADOS}

\section{Caracterização dos idosos diabéticos do PCA}

Dos 163 participantes do estudo, observouse predominância de pessoas do sexo feminino $(76,1 \%)$ e da faixa etária dos 60 aos 69 anos em ambos os sexos. O total de idosos com idade igual ou superior a 80 anos foi de apenas 6,1\%. Quanto ao estado civil, verificou-se entre os homens que a convivência com a esposa ou companheira representou $66,7 \%$ dos casos. Entre as mulheres, a condição de viuvez $(49,2 \%)$ teve maior representatividade. Verificou-se, ainda, que $35,5 \%$ das idosas e $23,1 \%$ dos homens moravam sozinhos no momento de sua inscrição no PCA.

Mesmo identificando que $79,9 \%$ das idosas foram classificadas como viúvas, separadas e solteiras, $64,5 \%$ moravam com alguém, geralmente, com pessoas do sexo masculino $(64,6 \%)$, sendo dois terços destes com idade superior a 18 anos. Essas companhias incluíam eventuais companheiros e, principalmente, filhos e netos. Entre os idosos, verificou-se que $66,1 \%$ das companhias foram pessoas do sexo feminino, sendo três quartos com idade superior a 18 anos. Nesse universo, estavam inseridas esposas, companheiras, filhas e netas. Quanto ao tempo de permanência no programa, identificou-se a mediana de 21,5 meses para as mulheres e de 16 meses para os homens. As especificações dos dados sociodemográficos estão apresentados na tabela 1 . 
Tabela 1 - Número e percentagem por faixa etária, estado civil, companhia de residência e composição familiar de idosos diabéticos do Programa Cidadania Alimentar. Canoas/RS, julho/2008.

\begin{tabular}{|c|c|c|c|c|c|c|}
\hline \multicolumn{7}{|c|}{ Sexo } \\
\hline \multirow{2}{*}{$\begin{array}{l}\text { Variável } \\
\text { Faixa Etária }\end{array}$} & \multicolumn{2}{|c|}{ Feminino } & \multicolumn{2}{|c|}{ Masculino } & \multicolumn{2}{|c|}{ Total } \\
\hline & $\mathrm{N}$ & $\%$ & $\mathrm{~N}$ & $\%$ & $\mathrm{~N}$ & $\%$ \\
\hline De 60 a 69 anos & 67 & 54,0 & 25 & 64,1 & 92 & 56,4 \\
\hline De 70 a 79 anos & 48 & 38,7 & 13 & 33,3 & 61 & 37,4 \\
\hline$\geq 80$ anos & 9 & 7,3 & 1 & 2,6 & 10 & 6,1 \\
\hline Total & 124 & 100,0 & 39 & 100,0 & 163 & 100,0 \\
\hline Estado Civil & $\mathrm{N}$ & $\%$ & $\mathrm{~N}$ & $\%$ & $\mathrm{~N}$ & $\%$ \\
\hline casado(a)/ c/ companh. & 25 & 20,2 & 26 & 66,7 & 51 & 31,3 \\
\hline viúvo(a) & 61 & 49,2 & 7 & 17,9 & 68 & 41,7 \\
\hline separado(a) & 27 & 21,8 & 5 & 12,8 & 32 & 19,6 \\
\hline solteiro(a) & 11 & 8,9 & 1 & 2,6 & 12 & 7,4 \\
\hline Total & 124 & 100,0 & 39 & 100,0 & 163 & 100,0 \\
\hline Com quem reside & $\mathrm{N}$ & $\%$ & $\mathrm{~N}$ & $\%$ & $\mathrm{~N}$ & $\%$ \\
\hline sozinho(a) & 44 & 35,5 & 9 & 23,1 & 53 & 32,5 \\
\hline com alguma companhia & 80 & 64,5 & 30 & 76,9 & 110 & 67,5 \\
\hline Total & 124 & 100,0 & 39 & 100,0 & 163 & 100,0 \\
\hline Composição Familiar & $\mathrm{N}$ & $\%$ & $\mathrm{~N}$ & $\%$ & $\mathrm{~N}$ & $\%$ \\
\hline adultos masculinos & 64 & 43,5 & 9 & 13,8 & 73 & 34,4 \\
\hline menores masculinos & 31 & 21,1 & 13 & 20,0 & 44 & 20,8 \\
\hline adultos femininos & 35 & 23,8 & 34 & 52,3 & 69 & 32,5 \\
\hline menores femininos & 17 & 11,6 & 9 & 13,8 & 26 & 12,3 \\
\hline Total & 147 & 100,0 & 65 & 100,0 & 212 & 100,0 \\
\hline
\end{tabular}

Na avaliação do IMC, para ambos os sexos e nas três categorias das faixas etárias, a população estudada apresentou medianas com valores superiores a $27 \mathrm{~kg} / \mathrm{m}^{2}$, o que caracteriza sobrepeso pelo parâmetro de Lipschitz. ${ }^{11}$ Do total dos idosos, 85 mulheres (68,5\%) e 39 homens (61,5\%) - apresentaram valores de IMC que indicaram sobrepeso. Os valores do IMC também foram maiores nas mulheres, comparativamente aos homens. A mediana dessa variável, em mulheres da faixa etária entre 60 e 69 anos, foi superior a 30 $\mathrm{kg} / \mathrm{m}^{2}$, o que caracteriza obesidade. ${ }^{12}$ Destas, 51 , estavam com o IMC superior a $27 \mathrm{~kg} / \mathrm{m}^{2}$, sendo que em 41 idosas o IMC foi superior a $30 \mathrm{~kg} / \mathrm{m}^{2}$. Esses resultados são apresentados na tabela 2. 
Tabela 2 - Valores medianos e respectivos desvios-padrão de IMC $\left(\mathrm{Kg} / \mathrm{m}^{2}\right)$, segundo sexo e grupos etários dos idosos diabéticos do Programa Cidadania Alimentar. Canoas, RS, julho/2008.

\begin{tabular}{lcccc}
\hline \multicolumn{1}{c}{ Faixa Etária } & Sexo & N & IMC $\left(\mathrm{Kg} / \mathrm{m}^{2}\right)$ & DP \\
\hline De 60 a 69 anos & Feminino & 67 & 31,6 & 5,5 \\
& Masculino & 25 & 28,9 & 2,9 \\
De 70 a 79 anos & Feminino & 48 & 27,7 & 4,7 \\
\multirow{3}{*}{$\geq 80$ anos } & Masculino & 13 & 27,0 & 2,7 \\
& Feminino & 9 & 29,8 & 3,2 \\
\hline
\end{tabular}

IMC - Índice de Massa Corporal

DP - Desvio Padrão

A condição de sobrepeso ou obesidade esteve presente em 70,1\% das mulheres e em 61,6\% dos homens estudados. Quanto à obesidade, encontra-se um quadro preocupante pelo fato dessa situação ser a condição de mais de $50 \%$ das mulheres investigadas. A classificação de magreza foi a que apresentou a menor frequência, apenas $4 \%$, e esteve presente somente entre as mulheres. A tabela 3 mostra a distribuição do estado nutricional dos idosos diabéticos do PCA mediante a classificação do IMC.

Tabela 3 - Distribuição dos idosos diabéticos do Programa Cidadania Alimentar, segundo sexo e classificação do IMC $\left(\mathrm{Kg} / \mathrm{m}^{2}\right)$. Canoas, RS, julho/2008.

\begin{tabular}{lrrrrrrrr}
\hline \multirow{2}{*}{ Classificação de IMC } & \multicolumn{3}{c}{ Sexo } & \multicolumn{2}{c}{ Masculino } & \multicolumn{2}{c}{ Total } \\
& \multicolumn{2}{c}{ Feminino } & \multicolumn{2}{c}{ N } & $\%$ & \multicolumn{2}{c}{ N } & $\%$ \\
\hline Magreza & $\mathrm{N}$ & $\%$ & $\mathrm{~N}$ & $\%$ & - & 5 & 3,1 \\
Eutrofia & 5 & 4,0 & - & - & 47 & 28,8 \\
Sobrepeso & 32 & 25,8 & 15 & 38,5 & 38 & 23,3 \\
Obesidade & 23 & 18,5 & 15 & 38,5 & 73 & 44,8 \\
\hline Total & 64 & 51,6 & 9 & 23,1 & 163 & 100,0 \\
\hline
\end{tabular}

IMC - Índice de Massa Corporal 
De posse dessa constatação, a mediana dos valores da NEE para as pessoas do sexo feminino foram superiores a 1.500 e inferiores a 1.700 quilocalorias (kcal) nos três grupos etários. Pelos mesmos critérios, os homens tiveram os valores superiores a 1.900 e inferiores a 2.200 kcal, conforme apresentado na tabela 4. Ficou constatado que, quanto maior a faixa etária, menor foi a NEE para cada grupo em ambos os sexos. Como limitação desta análise, deve ser salientado o fato de que nos prontuários não havia especificação para nível de atividade física dos idosos, sendo usado para isso o valor de 1,0 como coeficiente de atividade física.

Tabela 4 - Necessidades energéticas estimadas (NEE) dos idosos diabéticos e percentual de adequação em relação à composição da cesta básica diet do Programa Cidadania Alimentar. Canoas, RS, julho/2008.

\begin{tabular}{lccccc}
\hline \multicolumn{1}{c}{ Faixa Etária } & Sexo & N & NEE & DP & \% cbdiet \\
\hline De 60 a 69 anos & Feminino & 67 & 1687,8 & 141,5 & 54,8 \\
& Masculino & 25 & 2151,0 & 165,0 & 43,0 \\
De 70 a 79 anos & Feminino & 48 & 1603,4 & 122,3 & 57,6 \\
\multirow{3}{*}{$\geq 80$ anos } & Masculino & 13 & 2060,3 & 179,5 & 44,9 \\
& Feminino & 9 & 1512,2 & 139,5 & 61,1 \\
& Masculino & 1 & 1986,4 & - & 46,5 \\
\hline
\end{tabular}

DP - Desvio Padrão

Em relação à utilização de algum recurso para o controle de glicemia, os resultados foram bastante semelhantes entre a população masculina e feminina. A utilização de insulina pelos diabéticos do PCA foi de 15,4\% nos homens e $17,7 \%$ nas mulheres. Por sua vez, o uso de medicação hipoglicemiante estava registrado em $74,4 \%$ dos prontuários dos homens e $83,9 \%$ no das mulheres. Nos prontuários dos idosos diabéticos não havia informações ou registros sobre o nível glicêmico.

\section{Caracterização da cesta básica diet}

O Programa Cidadania Alimentar distribuiu no ano de 200816.725 cestas básicas para os idosos cadastrados, distribuídas nas seguintes composições: normal, 44,4\%; light, 43,6\%; e diet, $12 \%$. A cesta básica diet tem por composição os seguintes itens: arroz branco e integral, aveia em flocos, leite em pó desnatado, óleo de milho, feijão preto, massa com ovos, biscoito tipo cream-craker, adoçante à base de stévia e proteína texturizada de soja. A quantidade de cada tipo de alimento e dos respectivos valores calóricos com a distribuição dos carboidratos, proteínas, gorduras, fibras e sódio está apresentado no quadro 1. 
Quadro 1 - Composição e Valores Nutricionais da cesta básica diet do Programa Cidadania Alimentar. Canoas, RS, julho/2008.

\begin{tabular}{|c|c|c|c|c|c|c|c|}
\hline Produto & $\begin{array}{l}\text { Qtde. } \\
\text { (un) }\end{array}$ & $\begin{array}{l}\text { Valor } \\
\text { (kcal) }\end{array}$ & $\begin{array}{c}\text { Carboid. } \\
\text { (g) }\end{array}$ & $\begin{array}{l}\text { Prot. } \\
\text { (g) }\end{array}$ & $\begin{array}{l}\text { Gord. } \\
(\mathrm{g})\end{array}$ & $\begin{array}{c}\text { Fibra } \\
(\mathrm{g})\end{array}$ & $\begin{array}{l}\text { Sódio } \\
(\mathrm{Mg})\end{array}$ \\
\hline Arroz Branco & $1 \mathrm{~kg}$ & 3384 & 780 & 66 & 0 & 10 & 144 \\
\hline Arroz Integral & $1 \mathrm{~kg}$ & 3498 & 760 & 74 & 18 & 18 & 102 \\
\hline Aveia em Flocos & $250 \mathrm{~g}$ & 948,3 & 150 & 30,8 & 25 & 25 & 0 \\
\hline Leite em Pó Desnatado & $800 \mathrm{~g}$ & 2816 & 440 & 264 & 0 & 0 & 4000 \\
\hline Óleo de Milho & $900 \mathrm{ml}$ & 7477 & 0 & 0 & 830,8 & 0 & 0 \\
\hline Feijão Preto & $1 \mathrm{~kg}$ & 3247,3 & 583,3 & 200 & 12,7 & 216,7 & 900 \\
\hline Massa com ovos Espaguete & $500 \mathrm{~g}$ & 1736,9 & 362,5 & 56,3 & 6,9 & 13,8 & 125 \\
\hline Biscoito tipo Cream-Craker & $800 \mathrm{~g}$ & 3413,2 & 506,7 & 106,7 & 106,7 & 34,7 & 5493,3 \\
\hline Adoçante Stévia & $100 \mathrm{~g}$ & 360 & 90 & 0 & 0 & 0 & 0 \\
\hline Proteína texturizada de soja & $250 \mathrm{~g}$ & 850 & 81,2 & 131,2 & 0 & 50 & 0 \\
\hline Total & & 27730,7 & 3753,7 & 929,0 & 1000,1 & 368,2 & 10764,3 \\
\hline Valor diário & & 924,4 & 125,1 & 31,0 & 33,3 & 12,3 & 358,8 \\
\hline \multicolumn{3}{|c|}{ Distribuição \% de macronutrientes } & 54,2 & 13,4 & 32,5 & & \\
\hline
\end{tabular}

Avaliando a distribuição percentual dos macronutrientes, foram observados os seguintes valores: $54,2 \%$ de carboidratos, $13,4 \%$ de proteínas e 32,5\% de gorduras. Quanto ao valor energético, a composição diet fornece $924,4 \mathrm{kcal}$ por dia, considerando para isso o consumo de todos os itens disponibilizados. Os valores nutricionais das embalagens dos alimentos em relação aos resultados das tabelas de composição nutricional do IBGE e TACO foram inferiores a $5 \% .^{14,15}$

Os principais fornecedores de carboidratos na composição da cbdiet são os cereais arroz e aveia; os derivados de farináceos: biscoito tipo cream-craker e massa espaguete e o feijão. As proteínas da composição alimentar são de duas origens: animal, fornecida pelo leite em pó desnatado; e vegetal, cujas principais fontes são o feijão, a proteína texturizada de soja e o biscoito tipo cream-craker. As gorduras fornecidas estão concentradas no óleo de milho e também no biscoito tipo cream-craker, que foi o único produto que apresentava a gordura transaturada em sua composição.
As fibras fornecidas na cbdiet são classificadas principalmente como insolúveis que auxiliam no controle da saciedade, perda de peso e melhor funcionamento intestinal, e têm como principais fontes o feijão, a aveia, o biscoito tipo cream-craker e o arroz integral. Em relação aos teores de sódio, dois alimentos fornecidos concentram $88,2 \%$ do total desse mineral: o biscoito tipo cream-craker e o leite em pó desnatado.

Sobre o percentual de contribuição, o valor energético médio diário de 924,4 kcal da cbdiet supre de 54,8 a $61,1 \%$ das NEEs para as mulheres e de 43 a $46,5 \%$ para os homens (considerando a mediana por faixa etária em cada sexo). As especificações desses resultados foram apresentadas na tabela 4.

\section{DISCUSSÃO}

A saúde das pessoas não é um fenômeno isolado e envolve questões de caráter social, especialmente relacionadas às condições de vida, gênero, etnia e raça. Dos dados 
sociodemográficos apurados nos prontuários, algumas características merecem uma avaliação mais detalhada. A elevada proporção da população feminina se assemelha com outros estudos que mostram a maior participação de mulheres nos serviços e no desencadeamento de ações de promoção da saúde. ${ }^{16,17}$

Em relação ao estado civil, nos homens idosos do PCA, a característica de apresentar a companhia de uma esposa ou parceira é similar aos resultados encontrados no estudo Saúde, Bem-estar e Envelhecimento (SABE). ${ }^{16}$ Em contrapartida, entre as mulheres, a condição de viuvez representou praticamente metade dos casos, enquanto o fato de viver com um companheiro correspondeu a 20,1\%; situação essa bem diferente do verificado entre as idosas do estudo mencionado. ${ }^{16}$

Quanto à necessidade de companhia, os idosos do PCA têm diversos familiares que convivem com eles. Apesar de o programa considerar a composição da cbdiet exclusiva para o beneficiário cadastrado, a presença de mais pessoas numa mesma residência provavelmente interfere no consumo dos produtos disponibilizados para o idoso. A ausência de registro nos prontuários dos idosos sobre raça/cor autorreferida e escolaridade impossibilitou a comparação dessas variáveis. Entretanto, a pesquisa de Barceló et al. revelou que a diabetes em idosos esteve associada ao baixo nível de escolaridade. ${ }^{18}$

Apesar de o PCA ter um enfoque assistencial para os idosos considerados em situação de vulnerabilidade social, o tempo de permanência verificado foi bastante alto, confirmando, assim, uma dependência dos participantes em relação ao benefício. O prazo estipulado de 24 meses para a permanência no programa objetiva oportunizar que mais pessoas possam ter acesso a esse benefício. Entretanto, essa situação é questionável, uma vez que, na medida em que as pessoas vão ficando mais velhas, tendem a necessitar cada vez mais desse auxílio.

Para Lavinas et al., as políticas de alimentação e nutrição, apesar de bem intencionadas, acabam tendo uma conformação assistencialista por amenizar ou mascarar os reais efeitos de situações de exclusão social. ${ }^{19}$ Programas sociais, de qualquer natureza, sempre apresentam essa possibilidade de conotação assistencialista.

Ainda é importante ressaltar que nenhum programa público conseguirá atender plenamente a demanda de pessoas em condições de necessidade parcial ou total e que os esforços para garantir ou possibilitar uma vida mais digna a partir do fornecimento de alimentos é válida, e muitas vezes, fundamental à sobrevivência dos idosos. Nos prontuários do PCA, não há a obrigatoriedade de laudo médico confirmando o diagnóstico de DM. Essa situação impede a identificação de outros casos de idosos que poderiam ser diagnosticados como diabéticos entre os beneficiados das outras cestas básicas do PCA (versões normale light).

Os indicadores de sobrepeso e obesidade têm sido observados em vários estudos, nos quais pode ser verificado, especialmente entre as mulheres, que os valores de IMC superiores a $25 \mathrm{~kg} / \mathrm{m}^{2}$, têm atingido mais de $50 \%$ dessa população. ${ }^{17,20}$ Estudo em Curitiba (PR), com 1.069 mulheres idosas, identificou $43,5 \%$ delas com sobrepeso e 34\% em condição de obesidade. ${ }^{21}$ Barceló et al. verificaram forte associação da prevalência de diabetes com o IMC elevado. ${ }^{18}$ A condição de sobrepeso e obesidade também apresenta relação direta com a DM, pois no estudo de Gomes et al., ${ }^{22}$ realizado com 2.519 pacientes diabéticos, $75 \%$ da população estudada não se encontrava na faixa de peso ideal.

A prevalência do sobrepeso e obesidade parece ter associação direta com a idade, especialmente entre as mulheres. Estudos de Gigante et al. e Teichmann et al. confirmaram essa associação e encontraram percentuais superiores (41,5\% e $60 \%$ ) nas mulheres de mais idade, comparativamente às mais jovens. ${ }^{23,24}$ Está evidenciado também que a obesidade é predominante nas classes sociais menos favorecidas, estando associada ao padrão alimentar dessa população. ${ }^{23,25}$ Estudo de Lindström et al. apontaram que mudanças no estilo de vida, incluindo a redução do peso corporal associado à diminuição na ingesta calórica e de gorduras saturadas e no incremento 
no consumo de frutas e da atividade física, atuam na prevenção e na melhora do quadro dos pacientes com DM. ${ }^{26}$

Quanto às formas de controle da glicemia, o uso da medicação oral é a prática mais utilizada, seguida do uso de insulina pelos idosos diabéticos do PCA. No estudo SABE, ${ }^{16}$ o uso de medicamentos hipoglicemiantes foi referido por $64,3 \%$ dos idosos diabéticos enquanto o uso de insulina ficou em $12,7 \%$ dessa mesma população.

No início do programa, o PCA disponibilizava uma composição padronizada que deveria suprir $50 \%$ das necessidades energéticas de um idoso. Atualmente, o trabalho desenvolvido apresenta enfoque bastante diferenciado em relação ao seu início. As composições de cestas básicas normal, light e diet, em função das necessidades da população beneficiada, foram redefinidas. Dessa maneira, além do suprimento energético, as cestas básicas do PCA estão orientadas também para as situações das doenças crônicas encontradas entre os beneficiários, procurando oferecer alimentos que possam estar contribuindo para a melhoria das condições de saúde das pessoas.

Ao repensar as políticas públicas sobre alimentação e nutrição, Le Bihan et al. apresentam alguns fundamentos para uma nova abordagem da nutrição pública, na qual o direito à alimentação adequada contempla, entre outros: a garantia de qualidade nutricional da alimentação, respeitando culturas e preferências alimentares; o acompanhamento nutricional das populações, para a obtenção de dados estatísticos objetivos e para ajudar nas tomadas de decisão e a realização de pesquisa científica voltada para a solução dos problemas nutricionais da população. ${ }^{27}$

Em relação aos macronutrientes, a recomendação quanto aos percentuais de consumo segue algumas especificações. Os carboidratos devem ser a principal fonte de calorias na dieta, sendo que o consumo deve oscilar entre 55 a $65 \%$ do valor energético total de acordo com o IOM. ${ }^{13}$ Connor et al. sugerem de 60 a $70 \%$ a quantidade de carboidratos na dieta de pessoas diabéticas. ${ }^{28} \mathrm{~A}$ ingestão de proteínas na dieta deve variar de 0,8 a 1 grama por quilo de peso ideal para ambos os sexos ou o correspondente de 10 a $15 \%$ das NEEs. ${ }^{13,28} \mathrm{O}$ consumo de gorduras ou lipídios deve variar entre 20 e $35 \%$ do aporte energético da dieta, ${ }^{13,28}$ sendo que dentro desse percentual, uma terça parte ou menos deve ser de gorduras saturadas. Analisando esses aspectos, verifica-se que a composição da cesta básica diet apresenta a distribuição dos macronutrientes bem próxima das recomendações internacionais. No entanto, poderiam ser feitos alguns ajustes para aumentar o percentual de carboidratos e diminuir o de gorduras.

As recomendações da IOM sugerem o consumo que varia de 20 a 30 gramas de fibras por dia. ${ }^{13}$ Mesmo as principais fontes de fibras sendo alimentos como grãos integrais, frutas e vegetais diversos, a composição da cesta básica diet fornece, aproximadamente, metade do valor recomendado.

O sódio é encontrado, principalmente, no sal de cozinha. Dessa forma, faz-se importante o controle de consumo desse mineral. Connor et al. sugerem que o consumo diário não ultrapasse $2.400 \mathrm{mg} / \mathrm{dia}$, o que representa $6 \mathrm{~g}$ do cloreto de sódio (sal de cozinha). ${ }^{28}$ O IOM orienta a ingestão de 1,3g/dia de sódio, sendo que pode ser tolerado até 2,3 g/dia. ${ }^{13}$ Convém lembrar que muitos produtos industrializados utilizam o sal como conservante de alimentos, especialmente conservas e embutidos, os quais, mesmo não fazendo parte dos produtos da cbdiet, podem ser adquiridos e consumidos de outra forma. O percentual de sódio fornecido pelos alimentos da cbdiet corresponde a 29,6\% da recomendação diária.

\section{CONSIDERAÇÕES FINAIS}

Este estudo investigou a contribuição energética e dos macronutrientes da composição da cbdiet do Programa Cidadania Alimentar (PCA) com o estado nutricional dos idosos diabéticos. Os resultados mostraram que a composição da cbdiet fornecida pelo PCA fornece a média de $924,4 \mathrm{kcal} /$ dia, distribuída em $54,2 \%$ de carboidratos, $13,4 \%$ de proteínas e $32,5 \%$ 
de gorduras. Esses valores indicaram uma boa adequação energética da cesta básica diet, quando considerada a necessidade energética estimada (NEE) recomendada para os idosos.

O Programa Cidadania Alimentar, apesar de estar fornecendo uma composição específica de cesta básica para as necessidades da população diabética, precisará reavaliar suas estratégias e buscar alternativas para o enfrentamento da situação de sobrepeso identificada entre os idosos desse estudo.

Os conceitos da Estratégia Global para a Promoção da Alimentação Saudável, Atividade física e Saúde, da OMS, poderiam ser de grande utilidade nessa questão, auxiliando no desenvolvimento de programas de educação continuada, incluindo orientações sobre o uso adequado dos itens que compõem a cbdiet, além de outros alimentos e bebidas que façam parte da ingesta cotidiana do idoso.

As políticas públicas de segurança alimentar devem fazer constantes avaliações quanto à efetividade dos programas que se iniciam com a oferta, passam pela utilização, e devem considerar o real impacto sobre os indicadores de saúde e do estado nutricional da população beneficiada. ${ }^{29}$

\section{AGRADECIMENTOS}

Agradecemos aos professores, em especial Sheila Câmara e Maria Tereza Olinto, e à coordenação do PPG de Saúde Coletiva da Ulbra, pelo apoio. Também a Teresinha Sopram e Eloísa Gazzo, da Prefeitura de Canoas, por todo o suporte dado a este trabalho.

\section{REFERÊNCIAS}

1. Maluf RS, Menezes F, Valente FL. Contribuição ao tema da segurança alimentar no Brasil. Rev Cadernos de Debate. 1996;IV:66-68.

2. Brasil. Lei ${ }^{\circ} 11.346$, de 15 de setembro de 2006. Cria o Sistema Nacional de Segurança Alimentar e Nutricional - SISAN com vistas em assegurar o direito humano à alimentação adequada e dá outras providências.

3. World Health Organization. Growth of the Elderly population of the World. In: Health of the Elderly. Geneva: WHO, 1989. p. 8-31. (Technical Report Series, n. 779).

4. Conselho Nacional dos Secretários de Saúde. O desafio do modelo de atenção à saúde do SUS. In: SUS: avanços e desafios. Brasília: CONASS. 2006. 164 p.

5. Holman H, Lorig K. Patients as partners in managing chronic disease. Brit Med J. 2000;320:526-7.

6. Organização Mundial da Saúde. Cuidados inovadores para condições crônicas: componentes estruturais de ação. Brasília: 2003.

7. International Diabetes Federation [homepage da internet]. Diabetes epidemic out of control. [atualizado em 28 set 2008; acesso em 28 set 2008]. Disponível em: http://www.idf.org./home/ index.cfm? unode $=7 F 22 F 450-B 1 E D-43 B B-A 57 C$ B975D16A812D
8. Wild S, Roglic G, Green A, Sicree R, King H. Global Prevalence of Diabetes. Diabetes Care. 2004;27:1047-1053.

9. Coeli CM, Ferreira LGFD, Drbal MM, Veras RP, Camargo Jr KR, Cascão AM. Mortalidade em idosos por diabetes mellitus como causa básica e associada. Rev Saude Publica. 2002;36(2):135-40.

10. Federação das Associações de Municípios do Rio Grande do Sul [homepage da internet]. Municípios: Informações Municipais [atualizado em 23 ago 2008; acesso em 23 ago 2008]. Disponível em: http://ww2.famurs.com.br/informaçõesMunicipais? salaEntidade.php? exibeDadosGerais=1

11. Lipschitz DA. Screening for nutritional status in the elderly. Primary Care. 1994; 21(1):55-67.

12. World Health Organizarion [homepage da internet]. Global Database on Body Mass Index. [atualizado em 4 abr 2009; acesso em 4 abr 2009]. Disponível em: http://www.who.int/ bmi/index.jsp

13. Institute of Medicine [homepage da internet]. Dietary Reference Intakes of Energy, Carbohydrates, Fiber, Fat, Protein and Amino Acids (Macronutrients). Food and Nutrition Board. 2002. [atualizado em 4 abr 2009; acesso em 4 abr 2009]. Disponível em: http://www.fnic.nal.usda 
14. Instituto Brasileiro de Geografia e Estatística. Tabela de composição de alimentos / IBGE. 5. ed. Rio de Janeiro: IBGE 1999. 137 p.

15. Tabela brasileira de composição de alimentos - Versão II. Campinas: NEPA-UNICAMP, 2006. 105p.

16. Lebrão ML, Duarte YAO. Estudo Saúde, Bem-estar e Envelhecimento (SABE) 2000 e 2006. In: Estudo SABE [CD ROM]. São Paulo: Universidade de São Paulo; 2008.

17. Bueno JM, Martino HSD, Fernandes MFS, Costa LS, Silva RR, Avaliação nutricional e prevalência de doenças crônicas não transmissíveis em idosos pertencentes a um programa assistencial. Cien Saude Colet. 2008: 13(4):1237-1246.

18. Barceló A, Pelaez M, Rodriguez-Wong L, PastorValero M. The prevalence of diagnosed diabetes among the elderly of seven cities in Latin America and the Caribbean: The Health, Wellbeing, and Aging (SABE) Project. J Aging Health. 2006;18(2):224-239.

19. Lavinas L, Manão D, Garcia EH, Bittar M, Bahia M, Bezerra RA. Combinando compensatório e redistributivo: o desafio das políticas sociais no Brasil. Textos para Discussão no 748 . IPEA. Rio de Janeiro. 2000

20. Santos DM, Sichieri R. Índice de massa corporal e indicadores antropométricos de adiposidade em idosos. Rev Saude Publica. 2005; 39(2):163-68.

21. Buzzachera CF, Krause MP, Elsangedy HM, Hallage T, Granato P, Krinski K, et al. Prevalência de sobrepeso e obesidade geral e central em mulheres idosas da cidade de Curitiba, Paraná. Rev Nutr. 21(5):525-533, set/out., 2008.
22. Gomes MB, Gianella Neto D, de Mendonça E, Tambascia MA, Fonseca RM, Réa RR, et al. Prevalência de sobrepeso e obesidade em pacientes com diabetes mellitus do tipo 2 no Brasil: estudo multicêntrico nacional. Arq Bras Endocrinol Metab [online]. 2006, vol. 50, n. 1, p. 136-144. ISSN 0004-2730.

23. Gigante DP, Dias da Costa JS, Olinto MT, Menezes AMB, Macedo S. Obesidade da população adulta de Pelotas, Rio Grande do Sul, Brasil e associação com nível sócio-econômico. Cad Saude Publica. 2006 set: 22(9):1873-79.

24. Teichmann L, Olinto MT, Dias da Costa JS, Ziegler D. Fatores de risco associados ao sobrepeso e a obesidade em mulheres de São Leopoldo, RS. Rev Bras Epidemiol.2006; 9(3): 360-373

25. Monteiro CA, Benicio MH, Conde WL, Popkin BM. 2000b. Shifting obesity trends in Brazil. Eur J Clin Nutr. 2000 Apr;54(4):342-6.

26. Lindström J, Louheranta A, Mannelin M, Rastas M, Salminen V,Eriksson J, et al. The Finnish Diabetes Prevention Study. Diabetes Care. 2003 dec: 26 (12): 3230-36.

27. Le Bihan G, Delpeuch F, Maire B (org.). Alimentação, nutrição e políticas públicas. São Paulo, Instituto Pólis, 2003. 132 p. (Cadernos de Proposições para o Século XXI, 4).

28. Connor H, Annan F, Bunn E, Frost G, McGough $\mathrm{N}$, Sarwar T, et al. The implementation of nutritional advice for people with diabetes. Diabetes UK. Diabetic Med. 2003;20:786-807.

29. 29. Santos IS. Avaliação do impacto de programas nutricionais. Rev Nutr.22(1):141-150, jan.fev., 2009. 